\title{
Die individuelle Nutzenwahrnehmung aus dem summativen Validierungsverfahren der Weiterbildungsakademie Österreich im Spiegel struktureller und individueller Professionalisierung
}

\author{
Petra H. Steiner (D)
}

Eingegangen: 11. Juni 2018 / Angenommen: 17. Januar 2019 / Online publiziert: 6. Februar 2019 (C) Der/die Autor(en) 2019

Zusammenfassung Dieser Beitrag stellt Ergebnisse einer aktuellen Online-Befragung der Absolventinnen und Absolventen des Validierungsverfahrens der Weiterbildungsakademie (WBA) Österreich vor. Dazu werden Befragungsergebnisse quantitativ ausgewertet sowie Antworten auf offene Fragen qualitativ inhaltsanalytisch interpretiert. Die Ergebnisse zeigen vor dem Hintergrund der Konzepte zu individueller und struktureller Professionalisierung sowie bildungspolitischer Agenden, dass die WBA individuell und strukturell professionalisierend wirkt. Dies gelingt in besonders hohem Ausmaß durch die Vermittlung des Professionsbildes der Erwachsenenbildnerin bzw. des Erwachsenenbildners. Die Valorisierung der erworbenen Qualifikation aus dem summativen Validierungsverfahren bleibt mangelhaft. Durch das Durchlaufen der Zertifizierung werden Erkenntnisse und Reflexionen der Professionsangehörigen angestoßen, welche sie befähigen an der eigenen Professionalisierung mitzuwirken.

Schlüsselwörter Summatives Validierungsverfahren · Individuelle und strukturelle Professionalisierung · Individuelle Nutzenwahrnehmung · Erwachsenenbildner

The individual perception of validation procedures benefits: Evidence from the Austrian Academy of Continuing Education-Professionalisation in its individual and structure-related aspects

Abstract This article presents the results of a recent online survey of graduates of the Austrian Academy of Continuing Education. Methodically, the survey's results on benefit items are interpreted and answers to open questions are analyzed by

Mag. Dr. P. H. Steiner $(\bowtie)$

Weiterbildungsakademie Österreich, 1070 Wien, Österreich

E-Mail: petrasteiner@chello.at; petra.steiner@wba.or.at 
qualitative content analyses. Empirical findings are subject to an interpretation using concepts of professionalization and the political agenda of validation of informal and non-formal learning. An important finding is, that WBA enhances individual professionalization as well as a structure-related professionalization. Valorization of the newly acquired qualification on the labour-market is not attained. Gaining a new identity as adult educator and crucial knowledge concerning professionalization is the benefit which provides adult educators with the ability to work on their own professionalization.

Keywords Validation of competences - Summative approach · Individual and structure-related aspects of professionalization - Individual perception of benefits . Adult educator

\section{Einleitung}

Validierung von Kompetenzen stellt einen neuen Weg des Umgangs mit Lernen und Bewertung von Fähigkeiten dar und ist von daher ein neuer Gegenstand der Bildungsforschung. Vertrauen in ein derartiges Umdenken ist ein Schlüsselfaktor, aber auch wissenschaftliche Evidenz ist nötig, damit neue Verfahren nachhaltig wirken können (Werquin 2009, S. 21). Bildungspolitisch festgelegte Anliegen, die mit der Validierung non-formalen und informellen Lernens erreicht werden sollen, sind: Steigerung der Beschäftigungsfähigkeit und des Wirtschaftswachstums, Erleichterung von Mobilität von Arbeitskräften, bessere (Re-)Integration in den Arbeitsmarkt. Individuell soll die Motivation für lebensbegleitendes Lernen steigen, stärkeres Bewusstsein für Kompetenzen unabhängig vom Lernkontext sowie ein besseres Verständnis für den Wert von Bildung und Lernen erzielt werden. Des Weiteren soll das Nachholen von Bildungsabschlüssen leichter werden. Insbesondere und dezidiert gelten diese Ziele für benachteiligten Menschen (Bundesministerium für Bildung 2017, S. 9 und 10ff.; Europäische Union 2012; Cedefop 2017, S. 11).

Im vorliegenden Beitrag wird eine Evaluation der Weiterbildungsakademie Österreich (WBA) aufgearbeitet, die anlässlich ihres zehnjährigen Bestehens durchgeführt wurde (Weiterbildungsakademie Österreich 2017). Die WBA ist eine Kompetenzanerkennungs- und Zertifizierungsstelle speziell für Erwachsenenbildner und Erwachsenenbildnerinnen und ein „summativer Ansatz“, mit dem ,Zertifikate und Qualifikationen“ (Cedefop 2016a, S. 60) vergeben werden. Sie stellt ein Validierungsverfahren dar, welches (wie auch das SVEB-Zertifikat als ,autonomer Typus“, Schmid 2018) nicht zum formalen Bildungssystem gehört und seine Flexibilität aus dem Einbezug aller relevanten Akteure aus Bildung, Politik und der Arbeitswelt bezieht. ${ }^{1}$ Das WBA-Validierungsverfahren basiert auf standardisierten Qualifikations- und Kompetenzbeschreibungen sowie qualitativen und quantitativen Validierungskriterien. Kompetenzen sind lernergebnisorientiert definiert. Validierung findet mittels eines Methodenmixes von Materialanalyse, schriftlicher und mündlicher Be-

\footnotetext{
1 Biffl et al. (2012, S. 29f.) kategorisieren diese Form ohne Anschluss an das formale Bildungssystem als „formale Validierung“, welche neben ,formativer und summativer Validierung“ (ebd.) besteht.
} 
fragung sowie Beobachtung und dem „Mischverfahren“ Assessment-Center (Strauch et al. 2009, S. 41) statt. ${ }^{2}$

Analysiert man die Wirkungsweisen des WBA-Verfahrens insgesamt, so können zwei Aspekte getrennt werden: erstens Validierung von Kompetenzen allgemein und zweitens Professionalisierung von Erwachsenenbildnern. Beide Aspekte sind bereits untersucht. Die Validierungspraxis der WBA wird in Gugitscher und Schmidtke (2018) kritisch durchleuchtet und hinsichtlich ihrer pädagogischen Anlage und Validität untersucht sowie in Gruber (2018) beschrieben. Mehrmals wurde die WBA als Beispiel guter Validierungspraxis ausgewiesen (Cedefop 2010, S. $41 \mathrm{ff}$; EUCEN 2013). Als Professionalisierung vorantreibende Einrichtung ist das Verfahren seit Bestehen 2007 Gegenstand externer Evaluationen (Brünner et al. 2012; Brünner und Gruber 2014). Es wurde untersucht, ob die WBA zur „Professionalisierung und Qualitätssicherung in der Erwachsenenbildung/Weiterbildung“ beiträgt, was der wesentliche Grund zur Implementierung des Verfahrens war (Heilinger 2012). Weiterhin war die WBA auch mit dem speziellen Fokus der Überprüfung der Zielerreichung eines vom Europäischen Sozialfonds geförderten Projektes Gegenstand externer Evaluation (Steiner et al. 2014). Wenngleich das Verfahren jedoch mit bislang rund 1490 vergebenen beruflichen Abschlüssen (,WBA-Zertifikate und WBA-Diplome“) und nach beinahe zwölfjährigem Bestehen als erfolgreich gesehen werden kann (Weiterbildungsakademie Österreich 2018), lassen die Ergebnisse vorliegender Evaluationen erkennen, dass die Nachhaltigkeit ihrer Wirkung für Professionalisierung und Qualitätssicherung keineswegs gesichert ist. Nutzende der WBA sind motiviert und haben individuellen Gewinn zu verbuchen - im Sinne von Persönlichkeitsstärkung und Lernzugewinn, jedoch ist die Valorisierung (Geltendmachung des neuen beruflichen Abschlusses am Arbeitsmarkt der Erwachsenenbildner) nur mangelhaft gegeben. Letzteres jedoch wäre ein notwendiger Bestandteil „struktureller Professionalisierung“ (Kraus 2012, S. 39). Strukturelle Professionalisierung muss ,,individuelle Professionalisierung“ begleiten, sollen die professionellen Akteure Anreize zum Verbleib in der Berufssparte und zur Weiterbildung vorfinden (ebd.).

Mittels Befragungsergebnissen von 468 Absolventinnen und Absolventen des Verfahrens wird in diesem Beitrag untersucht, inwiefern das summative Validierungsverfahren individuelle und strukturelle Professionalisierung befördert, welchen Nutzen Absolventen generell wahrnehmen und - soweit das vorliegende Material dies ermöglicht - auch, ob sich die Ziele der bildungspolitischen Agenden (siehe weiter oben) in der Nutzenwahrnehmung der WBA-Absolventen spiegeln.

Methodisch gliedert sich die Analyse in zwei Teile: erstens eine quantitative Auswertung von 19 vorgegebenen Fragen der Evaluation und zweitens eine qualitative Inhaltsanalyse von offenen Kommentaren innerhalb dieser Befragung. Letztere dient dazu, die individuelle Perspektive abseits vorgegebener Fragen ins Zentrum zu stellen. Hier kann ein Stück weit geprüft werden, ob es für Absolventen hoch relevante Aspekte gibt, die weder in der Befragung noch in der strategischen Ausrichtung der WBA berücksichtigt sind.

2 Zur Beschreibung des Verfahrens der Weiterbildungsakademie Österreich siehe: Gruber (2017); Prokopp und Luomi-Messerer (2010); Weiterbildungsakademie Österreich (o.J.) 
Forschungsfragen sind:

1. Welchen Nutzen nehmen WBA-Absolventinnen und Absolventen wahr?

2. Inwiefern nehmen WBA-Absolventinnen und Absolventen Nutzen im Sinne individueller und struktureller Professionalisierung wahr? Valorisiert sich der Abschluss für WBA-Absolventen?

3. Gibt es Hinweise darauf, dass die WBA bildungspolitische Anliegen im Zusammenhang mit Kompetenzvalidierung realisiert (Beschäftigungsfähigkeit, Mobilität von Arbeitskräften, (Re-)Integration in den Arbeitsmarkt, Motivation für lebensbegleitendes Lernen, stärkeres Bewusstsein für Kompetenzen unabhängig vom Lernkontext, verbessertes Verständnis für den Wert von Bildung und Lernen, leichteres Nachholen von Bildungsabschlüssen)?

4. Welchen Nutzen abseits vorgegebener Kategorien und bildungspolitischer Intentionen nehmen die Absolventen wahr, was ist ihnen besonders wichtig?

\section{Ausgangslage, Forschungsergebnisse zum WBA-Verfahren}

Die WBA ist wissenschaftlich gut beschrieben und evaluiert (Gruber 2017). ${ }^{3} \mathrm{Im}$ Folgenden wird ein kurzer Einblick in bestehende Ergebnisse gegeben. In einer ersten Begleitstudie (Brünner et al. 2012) standen die Einführung des neuen Verfahrens, individuelle Erwartungen und Motive (ebd., S. 107) sowie insbesondere die institutionelle Trägerstruktur des Kooperativen Systems der Erwachsenenbildung in Österreich im Zentrum. In dieser und einer folgenden Begleitforschung wurde der Nutzen aus dem Validierungsverfahren breiter definiert (Brünner und Gruber 2014), indem die Trägerstruktur der Einrichtung in die Analyse einbezogen wurde (ebd., S. 4 ff.). Die zweite Phase dieser Begleitstudie war ähnlich angelegt und brachte die Ergebnisse, dass auf Ebene der erwachsenenbildnerischen Mitarbeitenden ein Professionalisierungsschub mithilfe des Verfahrens ausgelöst wurde (ebd., S. 111). Auf Ebene der Institutionen fanden sich weniger Auswirkungen, jedoch eine höhere Motivation für Weiterbildung (ebd., S. 112). Auf Ebene des gesamten Berufsfeldes stellten die Autorinnen wiederum hohe Wirksamkeit fest. Als Ergebnis wird hier auch eine ,idealtypische Kandidatin“ der WBA definiert, die auf Anerkennung bezüglich eines WBA-Abschlusses (z. B. Honorierung, aber auch mentale Anerkennung) seitens Dienstgebern Wert legt, sie strebt die Bilanzierung bislang erworbener Kompetenzen an und absolviert die WBA auch, um sich persönlich weiter zu entwickeln (ebd., S. 56f). In einer anderen Evaluation im Zuge der ESF-Förderüberprüfung (Steiner et al. 2014) wurde u. a. die hohe Zufriedenheit mit der Durchführung des Verfahrens festgestellt (ebd., S. 57) und die Motive für Zertifizierung erhoben. Es ergab sich bezüglich individueller Nutzenwahrnehmung das Ergebnis, dass der Nutzen bei persönlichen Aspekten hoch ist. Wenn es je-

\footnotetext{
3 Damit stellt sie eine Ausnahme dar. Denn da in vielen Ländern Europas die Implementierung von Validierungsangeboten erst im Gange ist, wird meist deren Umsetzung angeleitet und die Implementierung auf Ebene der Umsetzungsstrukturen überprüft. Viele vorliegende Studien vermitteln Basiswissen im Zusammenhang mit Validierung und geben einen Überblick über existierende Verfahren. Seltener wird deren Wirkung auf Individuen evaluiert. So meint auch das Cedefop (2016b, S. 41): ,evidence on outcomes and impact remains limited“.
} 
doch um die berufliche Verwertbarkeit (Position, Bezahlung) geht, so ist der Nutzen geringer (ebd., S. 42). Dabei sahen Nicht-Akademiker ihre Berufserfahrung durch das Verfahren stärker aufgewertet sowie sich stärker persönlich weiterentwickelt als Akademiker (ebd., S. 42f). Das verweist darauf, dass das Validierungsverfahren zur Stärkung von Gruppen führt, welche weniger Anerkennung auf Basis formaler Qualifikationen genießen als andere.

Die Ergebnisse der bisherigen Evaluationen zeigen insgesamt ein positives und teilweise gemischtes Bild: Das Verfahren selbst wird als gut und bereichernd wahrgenommen. In einer umfassenden, auch die Arbeitgeberseite einbeziehenden Perspektive auf Professionalisierung wird jedoch festgestellt, dass hier Anerkennung und Honorierung fehlen (ebd., S. 59) bzw. dass die breitere Anerkennung des Abschlusses des Validierungsverfahrens innerhalb der „Branche“ und von staatlicher Seite her ein ,work in process“ darstellt und hier bisher noch zu wenig erreicht wurde (Brünner und Gruber 2014, S. 60). Damit sind strukturelle Aspekte der Professionalisierung als unterentwickelt dargestellt. Die vorliegenden Studien unterscheiden bei ihren Ergebnissen zu „Professionalisierung“ jedoch nicht dezidiert individuelle und strukturelle Aspekte, das leistet der vorliegende Beitrag. Kritische Punkte der Nachhaltigkeit des Bestehens summativer Validierungsverfahrens kommen so klarer in den Blick.

\section{Definition von Begriffen und Konzepten}

Im Folgenden werden die wichtigsten Begriffe definiert und Konzepte kursorisch beschrieben.

- Kompetenz und Lernergebnisse: Unter Kompetenz wird die „Fähigkeit zur angemessenen Anwendung von Lernergebnissen in einem bestimmten Zusammenhang (Bildung, Arbeit, persönliche oder berufliche Entwicklung)“ (Cedefop 2014, S. 48) verstanden. Lernergebnisse sind „Aussagen darüber, was ein Lernender weiß, versteht und in der Lage ist zu tun, nachdem er einen Lernprozess abgeschlossen hat. Sie werden als Kenntnisse, Fertigkeiten und Kompetenzen definiert" (ebd., S. 74).

- Validierung von Kompetenzen und Lernergebnissen: Darunter versteht das Cedefop (ebd., S. 288): „Die Bestätigung durch eine zuständige Behörde oder Stelle, dass Lernergebnisse (Kenntnisse, Fähigkeiten und/oder Kompetenzen), die eine Person in einem formalen, nicht formalen oder informellen Kontext erzielt hat, gemäß festgelegten Kriterien bewertet wurden und den Anforderungen eines Validierungsstandards entsprechen. Die Validierung führt üblicherweise zur Zertifizierung."

- Summatives Validierungsverfahren: Das Cedefop (2016a, S. 60) definiert: „Summative Bewertungs- und Validierungsansätze dienen ausdrücklich der Formalisierung und Zertifizierung von Lernergebnissen und stehen in Bezug zu bzw. gehören zum Aufgabenbereich von Einrichtungen und Gremien, die zur Vergabe von Qualifikationen befugt sind. Formative und summative Bewertung sind jedoch nicht 
immer klar voneinander abzugrenzen, manchmal können Instrumente für beide Zwecke verwendet werden."

- Zertifizierung von Lernergebnissen: Die Zertifizierung von Lernergebnissen ist die „Ausstellung eines Zertifikats, Diploms oder Titels, das bzw. der formal bescheinigt, dass bestimmte Lernergebnisse (Kenntnisse, Know-how, Fähigkeiten und/ oder Kompetenzen), die eine Person erzielt hat, durch eine zuständige Behörde oder eine zuständige Stelle gemäß einem festgelegten Standard bewertet wurden“ (ebd., S. 15).

- Qualifikation: Qualifikation wird vom Cedefop (2014, S. 203) zweifach definiert: als das Ergebnis eines formalen Bildungsabschlusses und als Passung eines Abschlusses zu einem definierten Bedarf am Arbeitsmarkt: „Der Begriff Qualifikation umfasst verschiedene Aspekte: a) formelle Qualifikation: das formelle Ergebnis (Befähigungsnachweis, Bescheinigung, Diplom, Zertifikat, Zeugnis oder Titel) eines Bewertungsverfahrens. Im Rahmen dieses Verfahrens bestätigt eine zuständige Behörde oder Stelle, dass eine Person Lernergebnisse vorweisen kann, die sich an bestimmten Standards messen lassen, und/oder die notwendige Kompetenz besitzt, eine Aufgabe in einem bestimmten Tätigkeitsbereich auszuführen. Eine Qualifikation erkennt den Wert der Lernergebnisse am Arbeitsmarkt und in der allgemeinen und beruflichen Bildung offiziell an. Eine Qualifikation kann den rechtlichen Anspruch verleihen, einen bestimmten Beruf auszuüben (OECD); b) Anforderungen für einen Arbeitsplatz: Kenntnisse, Eignung und Fähigkeiten, die benötigt werden, um die spezifischen Aufgaben durchzuführen, die mit einem bestimmten Arbeitsplatz verbunden sind (ILO).“

\section{Strukturelle und individuelle Professionalisierung}

Zwei gleichzeitige Bewegungen bei Professionalisierung werden von Kraus (2012, S. 39) theoretisch als ,individuelle und strukturelle Professionalisierung“ gefasst.

Den gesellschaftlichen Aushandlungsprozess, die erreichten Merkmale einer Profession zu festigen und weitere zu erreichen, kann man als strukturelle Professionalisierung bezeichnen [...]. Dies beinhaltet neben Qualifizierungsaspekten und Formen der Selbstorganisation auch die Verstetigung von Erwerbschancen auf hohem Niveau, also die Konstitution eines professionellen Beschäftigungsfeldes.

Strukturelle Professionalisierung bedeutet unter anderem, dass es institutionalisierte Rahmungen für die Professionsausübung gibt, und dass die „Valorisierung“, d.h. die Bewertung einer durchlaufenen Kompetenzanerkennung am Arbeitsmarkt, hoch ist.

Strukturen bzw. strukturelle Elemente sind ein festgeschriebenes Berufsbild, offizielle Berufsbezeichnung(en), standardisierte Ausbildungen, gesetzliche Regelungen zur Gestaltung der Tätigkeit, berufliche Interessenvertretungen, festgeschriebene Gehaltsbedingungen, akademische Ausbildung für die Profession (Steiner 2018, S. $31 \mathrm{f}$.$) .$ 
Individuelle Professionalisierung wird dagegen im Individuum verortet und als „kompetentes pädagogisches Handeln, das sich auf Grundlagenwissen stützt und dieses durch Erfahrungen auswertet“" (ebd.) definiert. Im Gegensatz zu struktureller Professionalisierung ist der „Ort“ der Professionalisierung das Individuum an sich und es geht hier nicht um die Ausbildung von Strukturen und Institutionen, sondern um individuell verortbare Professionalität. ${ }^{4}$ Einseitige individuelle Professionalisierung bedeutet, dass viel Freiheit für Arbeitnehmende besteht, aber auch wenig Anbindung an Institutionen vorhanden ist. Der Zugang zu Arbeitsmärkten wird nicht oder nur wenig durch die Existenz von Institutionen unterstützt, welche der Profession und dem spezifischen Tätigkeitsbündel gewidmet sind. Nittel und Schütz (2013, S. 126) bezeichnen es als „Individualisierung“ von Professionalisierung, wenn Professionalisierung an Individuen abgegeben wird, ohne strukturelle Anreize wie bessere Verankerung und Stabilisierung von Erwerbschancen. Kosten, Ergebnisse und die Verantwortung würden hier einzelnen Erwachsenenbildenden überlassen (ebd.).

$\mathrm{Zu}$ ergänzen ist beim Konzept der strukturellen und individuellen Professionalisierung, dass beide Aspekte der Professionalisierung in einem Zusammenhang stehen und aufeinander verweisen. Sie stellen kein Entweder-Oder dar, sondern treten vielmehr beide gleichzeitig in unterschiedlichem Ausprägungsgrad auf.

\section{Valorisierung}

Das Konzept Valorisierung ist geeignet, Nutzenerwartungen aus summativen Validierungen zu kategorisieren. Valorisierung meint die „Geltendmachung der formalen oder non-formalen Qualifikationen in der Arbeitswelt“ (Heidemann und Kruse 1999, S. 40) und ist damit ein Teilaspekt struktureller Professionalisierung. Die Geltendmachung von Validierung ,kann im Betrieb eine höhere Einstufung in der Betriebshierarchie, ein Verantwortungs- bzw. Aufgabenzuwachs in der Stellendefinition oder auch eine verbesserte Entlohnung sein“ (Käpplinger 2002, S. 5 f.). Mit dem Begriff „Valorisierung“ wird die Perspektive der ,Verwertung von Qualifikation und Kompetenzen fokussiert“ (ebd.). Im deutschsprachigen Raum wird in der Regel nicht von „Valorisierung“, sondern lediglich von „Bewertung“ gesprochen.

\section{Erhebung, Datenbasis, Methodik}

Der Artikel arbeitet eine von Mai bis August 2017 durchgeführte Evaluation der WBA auf. Erfragt wurden online und anonym Alter, Geschlecht, Tätigkeitsprofil, Daten zur Beschäftigung in der Erwachsenenbildung, zu Motiven, die WBA zu durchlaufen, zu sonstigen Seminaren, die ergänzend besucht wurden und zum Nut-

\footnotetext{
4 Eine ähnliche Unterscheidung treffen Helsper und Tippelt (2011, S. 275). Sie unterscheiden Professionalisierung mit der Zielperspektive Profession, die vor allem auf die Gewinnung des Status einer Berufsgruppe oder Profession abzielt und Professionalisierung mit der Zielperspektive Professionalität, die auf eine hohe Kompetenz in der (individuellen) Ausübung einer beruflichen Tätigkeit abzielt.
} 
zen, den Absolventen aus der Validierung oder Teilen derselben gezogen hatten. ${ }^{5}$ Der Fragebogen umfasste 41 Fragen mit vorgegebenen Antwort-Items, zwei Fragen waren offen. Angeschrieben wurden 1059 Absolventen. Beantwortet wurde die Befragung von 468 Personen, die Rücklaufquote betrug damit 44,2\%. Der Zeitraum zwischen der Befragung und dem Abschließen des WBA-Verfahrens liegt bei rund $37 \%$ der Befragten zwischen 1 und 3 Jahren, bei einem Drittel der Befragten zwischen 4 und 6 Jahren und bei $20 \%$ der Befragten war der WBA-Abschluss mehr als 6 Jahre her. Damit umfassen die Antworten auch die „Langfristperspektive“ der Absolventen dieses summativen Validierungsverfahrens.

In diesem Beitrag erfolgt eine quantitative Analyse vorgegebener Frage-Items sowie eine qualitativ-inhaltsanalytische Betrachtung von offenen Antworten.

\subsection{Quantitative Analyse - Methode}

Grundhypothesen für die quantitative Analyse:

1. Die Nutzenwahrnehmung aus dem WBA-Validierungsverfahren bildet ab, dass die WBA sowohl individuelle Professionalisierung als auch strukturelle Professionalisierung vorantreibt. Individuelle Professionalisierung ist jedoch dominant, das bedeutet, dass die Wahrnehmung von Nutzen dort besonders stark ist, wo der Nutzen weniger mit der Veränderung von Strukturen einhergeht und auf individuelle Lernerfahrungen begrenzt ist.

2. WBA-Absolventen können ihre WBA-Zertifikate selten befriedigend valorisieren (=höhere Einstufung in der Betriebshierarchie, Verantwortungs- bzw. Aufgabenzuwachs in der Stellendefinition oder verbesserte Entlohnung). ${ }^{6}$

3. Es gibt Nutzen aus dem Validierungsverfahren, der bildungspolitische Intentionen spiegelt.

Methodisch erfolgt eine quantitative Auswertung der Fragen nach wahrgenommenen Nutzenfaktoren, welche mittels vorgegebener Items erfragt wurden (Möglichkeit der Beantwortung mit ,trifft voll zu/trifft eher zu/trifft eher nicht zu/trifft nicht zu“). Es wurden 19 Fragen von 41 ausgewählt, welche auf die Nutzenwahrnehmung des Validierungsverfahrens gesamt und nicht auf spezifische Aspekte wie zu besuchende Seminare oder das verpflichtende Assessment-Center zielten. Diese ,allgemeinen“ Items erlauben Aussagen zur Nutzenwahrnehmung. Sie fragen bspw. danach, ob ein positiver Effekt auf die berufliche Tätigkeit wahrgenommen wird, ob man beruflich motivierter ist, ob es monetäre Vorteile gibt, ob man persönlich profitiert hat.

Um die Antworten bezüglich der Fragestellung bewerten zu können, werden die 19 Frage-Items den Analysekategorien strukturelle Professionalisierung, Valorisierung, individuelle Professionalisierung und einzelnen Aspekte bildungspolitischer Intentionen im Bereich Validierung zugeordnet. Eine Zuordnung zu „strukturelle

\footnotetext{
5 Die Ausführungen zu Erhebung und Datenbasis der 10-Jahres-Evaluation sind entnommen aus Weiterbildungsakademie Österreich 2017, S. 4 ff. Die vorgegebenen Items wurden aus Teilbereichen des Verfahrens heraus entwickelt sowie aus den vorliegenden bislang durchgeführten Evaluationen abgeleitet. Die Auswertung ist unveröffentlicht.

6 Professionstheoretisch ist Valorisierung ein Teilaspekt struktureller Professionalisierung.
} 
Tab. 1 Übersicht über Frage-Items und zugeordnete Analysekategorien strukturelle Professionalisierung (Str. Prof.), Valorisierung, individuelle Professionalisierung (Ind. Prof.) und Motivation für lebensbegleitendes Lernen sowie leichteres Nachholen von Bildungsabschlüssen

\begin{tabular}{|c|c|}
\hline Frage & Zugeordnete Analysekategorie \\
\hline $\begin{array}{l}\text { Durch den wba-Abschluss haben sich meine beruflichen } \\
\text { Möglichkeiten erweitert (Zulassungen etc.) }\end{array}$ & Str. Prof. \\
\hline $\begin{array}{l}\text { Durch den wba-Abschluss habe ich bessere Chancen am } \\
\text { Arbeitsmarkt }\end{array}$ & Str. Prof. \\
\hline $\begin{array}{l}\text { Durch die wba konnte ich wichtige Kontakte und Netzwerke } \\
\text { aufbauen }\end{array}$ & Str. Prof. \\
\hline $\begin{array}{l}\text { Durch den wba-Abschluss hat sich meine berufliche Stellung } \\
\text { für mich positiv verändert (Dienstgeberwechsel, bessere } \\
\text { Position, interessanterer Tätigkeitsbereich, ...) }\end{array}$ & Str. Prof. \\
\hline $\begin{array}{l}\text { Hat sich Ihr wba-Abschluss positiv auf Ihr Gehalt bzw. } \\
\text { Honorar ausgewirkt? (dreiteilige Skala: „ja, deutlich/ja, ein } \\
\text { wenig/nein“) }\end{array}$ & $\begin{array}{l}\text { Str. Prof./ } \\
\text { Valorisierung }\end{array}$ \\
\hline $\begin{array}{l}\text { Ich habe mehr Einblick in die österreichische Erwachsenen- } \\
\text { bildungslandschaft }\end{array}$ & Ind. Prof. \& Aspekte str. Prof. \\
\hline $\begin{array}{l}\text { Ich sehe meine Stärken und Fähigkeiten als Erwachsenen- } \\
\text { bildner/in klarer }\end{array}$ & Ind. Prof. \& Aspekte str. Prof. \\
\hline Ich identifiziere mich stärker als Erwachsenenbildner/in & Ind. Prof. \& Aspekte str. Prof. \\
\hline $\begin{array}{l}\text { Der in Gang gesetzte Reflexionsprozess hat zu meiner Wei- } \\
\text { terentwicklung beigetragen }\end{array}$ & Ind. Prof. \\
\hline $\begin{array}{l}\text { Ich habe im Rahmen meines wba-Abschlusses zusätzliche } \\
\text { Kompetenzen erworben, die ich in meinem Berufsalltag gut } \\
\text { gebrauchen kann }\end{array}$ & Ind. Prof. \\
\hline Mein Selbstwert ist durch den wba-Abschluss gestiegen & Ind. Prof. \\
\hline $\begin{array}{l}\text { Meine berufliche Handlungskompetenz hat sich durch mei- } \\
\text { nen wba-Abschluss erweitert }\end{array}$ & Ind. Prof. \\
\hline $\begin{array}{l}\text { Durch den wba-Abschluss ist die Zufriedenheit mit meiner } \\
\text { beruflichen Tätigkeit gestiegen }\end{array}$ & Ind. Prof. \\
\hline Durch den wba-Abschluss bin ich beruflich motivierter & Ind. Prof. \\
\hline Ich nutze digitale Medien stärker als vor dem wba-Verfahren & Ind. Prof. \\
\hline $\begin{array}{l}\text { Durch meinen wba-Abschluss bin/war ich aufs Neue moti- } \\
\text { viert, mich weiterzubilden }\end{array}$ & $\begin{array}{l}\text { Motivation für lebensbegleitendes Ler- } \\
\text { nen steigt }\end{array}$ \\
\hline Mein Vertrauen in meine Lernfähigkeit ist gestiegen & $\begin{array}{l}\text { Motivation für lebensbegleitendes Ler- } \\
\text { nen steigt }\end{array}$ \\
\hline $\begin{array}{l}\text { Mein wba-Abschluss hat mich dazu motiviert, eine hoch- } \\
\text { schulische Ausbildung zu besuchen }\end{array}$ & $\begin{array}{l}\text { Motivation für lebensbegleitendes Ler- } \\
\text { nen steigt \& leichteres Nachholen von } \\
\text { Bildungsabschlüssen }\end{array}$ \\
\hline $\begin{array}{l}\text { Ich habe mehr Freude daran, mein Wissen mit anderen zu } \\
\text { teilen }\end{array}$ & $\begin{array}{l}\text { Motivation für lebensbegleitendes Ler- } \\
\text { nen steigt }\end{array}$ \\
\hline
\end{tabular}

Professionalisierung“ bedeutet, dass das Befragungsergebnis zu diesem Item darüber Auskunft gibt, wie sehr die WBA strukturelle Professionalisierung vorantreibt. Strukturelle Professionalisierung lässt sich erkennen, wenn im Item institutionalisierte organisierte Formen der Professionsausübung beinhaltet sind. Eine Zuordnung zu ,Valorisierung“ gibt Auskunft darüber, ob WBA-Absolventen monetäre oder berufspositionsbezogene Vorteile aus der Validierung ziehen konnten. Eine Zuordnung 
zu ,individuelle Professionalisierung“ bedeutet, dass das erfragte Item Kompetenzzuwachs auf individueller Ebene beinhaltet. Mitunter sind beide Formen in einem Frage-Item enthalten, etwa wenn nach der individuell zu verortenden ,Identität als ErwachsenenbildnerIn" gefragt wird. Individuell verortet ist hier die erlebte Identität, das Konstrukt „ErwachsenenbildnerIn“ jedoch hat bereits institutionalisierte/ strukturelle Elemente und ist an eine bestehende Definition gebunden. Bezüglich der Fragerichtung „Realisierung bildungspolitischer Intentionen“ kann zumindest für zwei bildungspolitische Ziele analysiert werden, ob die WBA hier einen Beitrag leistet, da diese vorhandenen Frage-Items zugeordnet werden können. Die Aspekte sind: Motivation für lebensbegleitendes Lernen und leichteres Nachholen von Bildungsabschlüssen.

In Tab. 1 findet sich ein Überblick über die Frage-Items und die Zuordnung zu den Analysekategorien strukturelle Professionalisierung, Valorisierung, individuelle Professionalisierung, Motivation für lebensbegleitendes Lernen, leichteres Nachholen von Bildungsabschlüssen.

\subsection{Qualitative Analyse - Methode}

Ein zweiter Analyseteil folgt methodisch der qualitativen Inhaltsanalyse nach Mayring und hier der Technik der Zusammenfassung und induktiven Kategorienbildung (Mayring 2010, S. 67 ff.). Nach der Auseinandersetzung mit individueller Wahrnehmung auf Basis vorgegebener Fragebogenitems sollen hier offene Antworten ohne vorgegebene Nutzenkategorien betrachtet werden. Methodologisch ist dieser Teil der vorliegenden Analyse explorativ und weitet den inhaltlichen Zugang zur subjektiven Wahrnehmung von Validierung durch Nutzende. Die Frage „Auf welche Aspekte abseits vorgegebener Kategorien legen Individuen wert, wenn sie ein Validierungsverfahren durchlaufen? " wird hier beantwortet. Womöglich stößt man hier auf Nutzen aus Validierungsverfahren, welcher über „Professionalisierung und Qualitätssicherung in der Erwachsenenbildung/Weiterbildung " und über die bildungspolitischen Ziele, die mit Validierung verfolgt werden, hinausgehen? Die individuelle Sicht auf Validierung soll stärker ins Zentrum der Aufmerksamkeit gerückt werden.

77 vollständige schriftliche Antworten auf die Frage ,Welche anderen Auswirkungen, die bisher nicht angesprochen wurden, haben Sie bei sich beobachtet? " wurden der Analyse unterzogen. Laut Mayring muss im Zuge der kommunikationswissenschaftlichen Analyse zuerst eine Kontextbestimmung erfolgen, die Auskunft über die Einbettung der kommunikativen Akte gibt. Danach wird die gesamte Auswertungseinheit festgelegt, danach wiederum die Kodiereinheit. Mittels eines mehrstufigen Abstrahierens von Aussagen (Paraphrasierung, Generalisierung auf ein Abstraktionsniveau, erste und zweite Reduktion) kommt man von konkreten Aussagen auf abstrahierende Kategorien, die das Zu-Analysierende zusammenfassen (vgl. ebd. S. $70 \mathrm{ff}$.): Der Kontext der hier analysierten Texte ist eine anonyme schriftliche Befragung von Absolventinnen und Absolventen der WBA. Die analysierten Aussagen wurden von den WBA-Absolventen auf die oben genannte Frage (Nr. 29) gegeben. Die qualitativ-inhaltsanalytischen Auswertungseinheiten sind 77 vollständige schriftliche Antworten im Fragebogen auf die genannte Frage. Die Länge dieser schriftlichen Aussagen liegt zwischen 2 und 36 Wörtern. Eine Kodiereinheit war 
entweder eine vollständige schriftliche Aussage oder ein inhaltlich abtrennbarer Aussagenteil, wobei eine vollständige Aussage von Absolventen maximal 3 Aussagenteile, also 3 Kodiereinheiten darstellt. Aus 77 vollständigen Antworten auf die gestellte Frage ergaben sich so 107 Kodiereinheiten.

\section{Ergebnisse der quantitativen Auswertung zur Wahrnehmung des Nutzens der WBA-Validierung}

In Tab. 2 wird die Nutzenwahrnehmung bei einzelnen Fragen dargestellt. Der Anteil der positiven Antworten mit ,trifft zu/trifft eher zu“ ist ausgewiesen. Weiters sind Analysekategorien zu den Frage-Items zugeordnet.

Ersichtlich wird, dass weniger als die Hälfte der WBA-Absolventen Nutzen im Bereich strukturelle Professionalisierung wahrnehmen. Besonders hoch ist die Ablehnung der Aussage, dass eine positive Veränderung der beruflichen Stellung (bessere Position, bessere Tätigkeit) stattfände und dass sich der erworbene Abschluss positiv auf das Gehalt oder Honorar auswirke. Die Valorisierung eines WBA-Abschlusses ist dieser Befragung nach kaum möglich.

Es gibt Frage-Items, die gleichzeitig individueller und struktureller Professionalisierung zuzuordnen sind (sie enthalten die strukturellen Elemente Professionsbild, Disziplinzugehörigkeit). Genau hier und nur bei diesen Fragen zeigt sich besonders ausgeprägt, dass die WBA nicht nur individuell, sondern auch strukturell professionalisierend wirkt. Die Stärke liegt in der Vermittlung eines Professionsbildes (,ErwachsenenbildnerIn“ und „Erwachsenenbildungslandschaft“), die Definition einer Profession ist ein Bestandteil struktureller Professionalisierung. Bei weit mehr als der Hälfte der Absolventen ist dieses strukturelle Element angekommen.

Im Bereich der rein individuellen Professionalisierungsaspekte wiederum zeigt sich, dass v. a. das Anregen von Reflexionsprozessen und das Anstoßen von Weiterentwicklung ein großer Nutzen ist (bei 71,5-78,1\% der Befragten). Weitere Aspekte der individuellen Professionalisierung erzielen ebenfalls höhere Werte als die strukturelle Professionalisierung. Etwa die Hälfte der Befragten hat Kompetenzen erworben, die im Berufsalltag gut brauchbar sind, der Selbstwert ist bei etwa der Hälfte gestiegen.

Bei den Frage-Items, welche Auskunft über die bildungspolitischen Ziele geben, zeigt sich, dass um die Hälfte der Befragten in dieser Hinsicht profitieren. Lediglich das Nachholen von Bildungsabschlüssen - hier eine hochschulische Ausbildung ist bei nur 15,2-21,1\% der Befragten angeregt worden.

So lässt sich zusammengefasst sagen, dass die Hypothesen (siehe oben) bestätigt wurden. Die WBA wirkt individuell professionalisierend sowie strukturell professionalisierend. Die individuelle Professionalisierung ist jedoch viel stärker ausgeprägt. Die Valorisierung der Qualifikation, ein Teil struktureller Professionalisierung, ist mit etwa $10 \%$ der Absolventen, die dies erreichten, äußerst gering ausgeprägt. Bei den strukturellen Aspekten von Professionalisierung war v.a. die Vermittlung eines Professionsbildes und einer Vorstellung von der „Erwachsenenbildungslandschaft“ relevant. Bei individueller Professionalisierung v.a. die Weiterentwicklung durch das Anregen von Reflexion. 
Tab. 2 Anteil positiver Antworten (,trifft zu, trifft eher zu“) bei 19 Frage-Items und Analysekategorien strukturelle Professionalisierung (Str. Prof.), Valorisierung, individuelle Professionalisierung (Ind. Prof.), Motivation für lebensbegleitendes Lernen sowie leichteres Nachholen von Bildungsabschlüssen

\begin{tabular}{|c|c|c|c|c|c|}
\hline Frage & Anteil & $\begin{array}{l}\text { Konf.- } \\
\text { Int. } \\
\text { Untere } \\
\text { Grenze }\end{array}$ & $\begin{array}{l}\text { Konf.- } \\
\text { Int. } \\
\text { Obere } \\
\text { Grenze }\end{array}$ & $n=$ & $\begin{array}{l}\text { Analyse-kate- } \\
\text { gorie }\end{array}$ \\
\hline $\begin{array}{l}\text { Durch den wba-Abschluss ha- } \\
\text { ben sich meine beruflichen } \\
\text { Möglichkeiten erweitert (Zulas- } \\
\text { sungen etc.) }\end{array}$ & 45,45 & 41,68 & 49,28 & 462 & Str. Prof. \\
\hline $\begin{array}{l}\text { Durch den wba-Abschluss } \\
\text { habe ich bessere Chancen am } \\
\text { Arbeitsmarkt }\end{array}$ & 43,65 & 39,85 & 47,53 & 449 & Str. Prof. \\
\hline $\begin{array}{l}\text { Durch die wba konnte ich wich- } \\
\text { tige Kontakte und Netzwerke } \\
\text { aufbauen }\end{array}$ & 31,67 & 28,22 & 35,33 & 461 & Str. Prof. \\
\hline $\begin{array}{l}\text { Durch den wba-Abschluss hat } \\
\text { sich meine berufliche Stel- } \\
\text { lung für mich positiv verändert } \\
\text { (Dienstgeberwechsel, bessere } \\
\text { Position, interessanterer Tätig- } \\
\text { keitsbereich, ...) }\end{array}$ & 20,92 & 17,97 & 24,20 & 459 & Str. Prof. \\
\hline $\begin{array}{l}\text { Hat sich Ihr wba-Abschluss } \\
\text { positiv auf Ihr Gehalt bzw. } \\
\text { Honorar ausgewirkt? (dreitei- } \\
\text { lige Skala: „ja, deutlich/ja, ein } \\
\text { wenig/nein“) }\end{array}$ & 9,95 & 7,80 & 12,61 & 464 & $\begin{array}{l}\text { Str. Prof., } \\
\text { Valorisierung }\end{array}$ \\
\hline $\begin{array}{l}\text { Ich habe mehr Einblick in die } \\
\text { österreichische Erwachsenen- } \\
\text { bildungslandschaft }\end{array}$ & 79,69 & 76,43 & 82,61 & 458 & $\begin{array}{l}\text { Ind. Prof. \& } \\
\text { Aspekte str. } \\
\text { Prof. }\end{array}$ \\
\hline $\begin{array}{l}\text { Ich sehe meine Stärken und } \\
\text { Fähigkeiten als Erwachsenen- } \\
\text { bildner/in klarer }\end{array}$ & 77,78 & 74,43 & 80,80 & 459 & $\begin{array}{l}\text { Ind. Prof. \& } \\
\text { Aspekte str. } \\
\text { Prof. }\end{array}$ \\
\hline $\begin{array}{l}\text { Ich identifiziere mich stärker als } \\
\text { Erwachsenenbildner/in }\end{array}$ & 69,06 & 65,41 & 72,49 & 459 & $\begin{array}{l}\text { Ind. Prof. \& } \\
\text { Aspekte str. } \\
\text { Prof. }\end{array}$ \\
\hline $\begin{array}{l}\text { Der in Gang gesetzte Refle- } \\
\text { xionsprozess hat zu meiner } \\
\text { Weiterentwicklung beigetragen }\end{array}$ & 75,00 & 71,54 & 78,17 & 460 & Ind. Prof. \\
\hline $\begin{array}{l}\text { Ich habe im Rahmen meines } \\
\text { wba-Abschlusses zusätzliche } \\
\text { Kompetenzen erworben, die } \\
\text { ich in meinem Berufsalltag gut } \\
\text { gebrauchen kann }\end{array}$ & 53,04 & 49,21 & 56,84 & 460 & Ind. Prof. \\
\hline $\begin{array}{l}\text { Mein Selbstwert ist durch den } \\
\text { wba-Abschluss gestiegen }\end{array}$ & 52,98 & 49,12 & 56,81 & 453 & Ind. Prof. \\
\hline $\begin{array}{l}\text { Meine berufliche Handlungs- } \\
\text { kompetenz hat sich durch mei- } \\
\text { nen wba-Abschluss erweitert }\end{array}$ & 48,92 & 45,12 & 52,73 & 464 & Ind. Prof. \\
\hline $\begin{array}{l}\text { Durch den wba-Abschluss ist } \\
\text { die Zufriedenheit mit meiner } \\
\text { beruflichen Tätigkeit gestiegen }\end{array}$ & 37,00 & 33,36 & 40,80 & 454 & Ind. Prof. \\
\hline
\end{tabular}


Tab. 2 (Fortsetzung)

\begin{tabular}{|c|c|c|c|c|c|}
\hline Frage & Anteil & $\begin{array}{l}\text { Konf.- } \\
\text { Int. } \\
\text { Untere } \\
\text { Grenze }\end{array}$ & $\begin{array}{l}\text { Konf.- } \\
\text { Int. } \\
\text { Obere } \\
\text { Grenze }\end{array}$ & $n=$ & $\begin{array}{l}\text { Analyse-kate- } \\
\text { gorie }\end{array}$ \\
\hline $\begin{array}{l}\text { Durch den wba-Abschluss bin } \\
\text { ich beruflich motivierter }\end{array}$ & 34,94 & 31,37 & 38,70 & 455 & Ind. Prof. \\
\hline $\begin{array}{l}\text { Ich nutze digitale Medien stär- } \\
\text { ker als vor dem wba-Verfahren }\end{array}$ & 11,01 & 8,82 & 13,66 & 454 & Ind. Prof. \\
\hline $\begin{array}{l}\text { Durch meinen wba-Abschluss } \\
\text { bin/war ich aufs Neue motiviert, } \\
\text { mich weiterzubilden }\end{array}$ & 47,95 & 44,15 & 51,77 & 463 & $\begin{array}{l}\text { Motivation für } \\
\text { Lernen steigt }\end{array}$ \\
\hline $\begin{array}{l}\text { Mein Vertrauen in meine Lern- } \\
\text { fähigkeit ist gestiegen }\end{array}$ & 45,97 & 42,18 & 49,81 & 459 & $\begin{array}{l}\text { Motivation für } \\
\text { Lernen steigt }\end{array}$ \\
\hline $\begin{array}{l}\text { Ich habe mehr Freude daran, } \\
\text { mein Wissen mit anderen zu } \\
\text { teilen }\end{array}$ & 39,00 & 35,33 & 42,80 & 459 & $\begin{array}{l}\text { Motivation für } \\
\text { Lernen steigt }\end{array}$ \\
\hline $\begin{array}{l}\text { Mein wba-Abschluss hat mich } \\
\text { dazu motiviert, eine hochschuli- } \\
\text { sche Ausbildung zu besuchen }\end{array}$ & 17,98 & 15,22 & 21,13 & 456 & $\begin{array}{l}\text { Motivation für } \\
\text { Lernen steigt } \\
\& \text { Nachholen } \\
\text { von Bildungs- } \\
\text { abschlüssen } \\
\text { erleichtert }\end{array}$ \\
\hline
\end{tabular}

Irrtumswahrscheinlichkeit $10 \%$

Berechnungen mit „R“ durchgeführt

Die WBA hat auch eine Wirkung im Bereich der bildungspolitischen Ziele, die mit Kompetenzvalidierung verbunden sind. Sie motiviert sich weiterzubilden, generiert Vertrauen in eigene Lernfähigkeit, sie regt aber weniger zum Besuch einer Hochschule an, damit stößt sie auf dieser gehobenen Ebene keine weiteren Bildungsgänge an.

\section{Ergebnisse der qualitativen Analyse zur Wahrnehmung des Nutzens der WBA-Validierung}

Die qualitative Analyse zeigt auf, welche Aspekte die Absolventen dieses Zertifizierungsverfahrens als so relevant erachteten, dass sie es nach der Befragung nach diversen Benefits aus Teilen des Verfahrens nochmals erwähnen. Einige Ergebnisse zeigen, dass die Nutzenwahrnehmung der Absolventen über vorgegebene Fragen und über institutionelle Intentionen der WBA hinausgehen.

Im Folgenden werden die induktiv gebildeten Kategorien aus der qualitativen Inhaltsanalyse vorgestellt. Innerhalb einzelner Kategorien können sich sowohl positive als auch negative Bewertungen und Haltungen finden, etwa wenn zur akademischen Wissensbasis einerseits sehr positive und andererseits sehr kritische Wahrnehmungen geäußert werden. Genauere Analysen über diese Nutzensicht der Absolventen wären äußerst interessant. Im Folgenden werden als ein erster Schritt die 12 inhaltlichen Aspekte genannt, welche Absolventen wichtig waren; dabei wird jeweils ein beispielhaftes Zitat zugeordnet: 
- Berufspraktische Hilfe durch Wissen: Absolventen erachten als relevant, dass sie Wissen erworben haben, welches sich im Arbeitsalltag umsetzen lässt: „,WBAAbschluss, Erfahrung und Kennenlernen der Institution unterstützt mich bei der Beratung anderer Menschen ".

- Berufspraktische Hilfe für die Positionierung am Arbeitsmarkt: Das Verfahren wird hinsichtlich seiner Möglichkeiten behandelt, Absolventen am Arbeitsmarkt zu stärken, insofern, als es Instrumente dazu an die Hand gibt, etwa durch das Zertifikat oder das Logo, das sie verwenden dürfen. So sagt eine befragte Person: „Ich habe überhaupt meinen Job erhalten. Das WBA-Zertifikat war eine Bedingung für die Einstellung. Vorteil: Gäbe es die WBA nicht, wäre ich vielleicht mit meinem bunten Qualifikationsmix nicht aufgenommen worden. “

- Reaktion und Relevanzen am Erwachsenenbildungs-Arbeitsmarkt: In dieser Kategorie finden sich Äußerungen dazu, wie der Arbeitsmarkt den WBA-Abschluss honoriert. In der inhaltlichen Aussage gehen die Stellungnahmen hier von ,wird angenommen und gebraucht" bis ,hat keine Relevanz, wird überhaupt nicht registriert": „Im Nachhinein Zorn, weil bisher nie jemand nach meinem WBA Abschluss gefragt hat", „für den Fortbestand des aktuellen Dienstverhältnisses war die Zertifizierung wichtig, und wurde vorausgesetzt. “

- Berufliche Identität ErwachsenenbildnerIn: Ein Relevanzfaktor ist die eigene Identität und die Auseinandersetzung mit sich selbst als Erwachsenenbildnerin: „Ich bezeichne mich mit Selbstsicherheit als Andragogin. “

- Erwachsenenbildung als Berufsbereich/Profession: Zusätzlich zur Identität als Erwachsenenbildnerin taucht die Kategorie Erwachsenenbildung als Profession auf. Viele Aussagen belegen, dass die Erwachsenenbildung erstmals besser kennengelernt wurde. Einige üben auch Kritik an diesem Teil des Bildungswesens, andere wiederum erleben Bereicherung: „Für jemanden, der aus der unmittelbaren Praxis des naturwissenschaftlich-technischen Wissenstransfers kommt, ist es ein großer Zugewinn, im Rahmen der Zertifizierung den Horizont in Richtung theoretischer Überbau der Erwachsenenbildung, Strukturen etc. [zu erweitern, Anm. P.H. Steiner]. “

- Auseinandersetzung mit akademischer Wissensbasis: Theoriewissen und seine Bedeutung ist eine relevante Kategorie in der Wahrnehmung der Absolventen. Dabei sehen die Befragten dieses entweder kritisch oder affirmativ oder sie stellen den Erwerb desselben schlicht fest. Eine befragte Person meint, sie sei „kritischer gegenüber Pädagogik als Hilfestellung in der EB“.

- Persönliche Stärkung und Reifung, soziales Lernen: Diese Kategorie zeigt an, dass der persönliche Gewinn, die Bestätigung, das Lob, der Gewinn von Selbstvertrauen ein Relevanzfaktor ist. In diese Kategorie fällt auch die Bestätigung eigener (praktischer) Fähigkeiten, die bisher Gegenstand von Selbstkritik oder Selbstzweifel waren. Durch das Durchlaufen der WBA werden Selbstzweifel aufgehoben oder gemindert: „, Große Zufriedenheit, dass die Summe meiner vorherigen einzelnen Weiterbildungen einen positiven, bestätigten Wert hat. “

- Stärkung der Durchsetzungsfähigkeit: In eine ähnliche Kerbe schlägt dieser Relevanzfaktor, der betont, dass durch das Durchlaufen der WBA mehr Durchsetzungsfähigkeit gegen Anforderungen, die nicht als gerechtfertigt angesehen wer- 
den, besteht. Zusätzlich zur allgemeinen Stärkung der Person wird hier betont, sich besser wehren zu können: „Ich positioniere mich stärker gegen plötzliche verpflichtende Ausbildungen, die von Fördergebern plötzlich konstruiert werden. “

- Diskussion des Bildungssystems: Das Bildungssystem und dessen Betrachtung ist ein Relevanzfaktor der Wahrnehmung. Einerseits wurde hier mehr Wissen und Einsicht gewonnen: „Ich sehe und beobachte den Ausbildungs- und Weiterbildungsmarkt bewusster. “ Andererseits wird auch Kritik am System geäußert: „Bin kritischer in meiner Haltung gegenüber Bildungsgeschehen und Bildungssystem geworden."

- Zurückweisung der Erwartung, Neues erlebt zu haben oder Austritt: Eine eigene Kategorie bilden Aussagen, die Ausstieg aus dem Tätigsein in der Erwachsenenbildung benennen, etwa: „Mir fehlt die Motivation, auf dem Gebiet besser $z u$ werden, daher bin ich heute nicht mehr in der Erwachsenenbildung tätig. “ Ebenfalls in diese Kategorie fallen Aussagen, die zurückweisen, wegen des Validierungsverfahrens mehr oder anderes gelernt zu haben: „Alle oben genannten Punkte von 20 bis 29 waren bereits vor dem WBA-Zertifikat enorm ausgeprägt. Ich war immer motiviert und mir meiner Stärken bewusst [kein Beitrag durch WBA] “.

- Ich und Lernen: In diese Kategorie fällt die Relevanz von Anschlusslernen, das eigene lernende oder auch lebenslang lernende Ich wird thematisiert: „Es war ein schönes, angstfreies, anspornendes Lernen."

- Verfahren Kompetenzanerkennung: Das Verfahren selbst ist eine Relevanzkategorie in der Wahrnehmung. Absolventen sehen es als relevant, das Verfahren und die Logik der Kompetenzanerkennung besser zu kennen. Sie empfehlen es weiter oder verwenden auch Kritik auf einzelne Teile des Verfahrens: „Ich habe das System der WBA verstanden und kann es weiterempfehlen. “ Oder auch: „Das Wissen, das ich erwarb, ist schon wieder weg. "

Vergleicht man die induktiv gebildeten Inhaltskategorien mit der quantitativen Erhebung, so wiederholt sich hier vieles (Nutzen für Chancen am Arbeitsplatz beispielsweise). Auch zeigt sich hier nochmals, dass individuelle und strukturelle Professionalisierung durch das summative Validierungsverfahren gelingt. Über die quantitativ erhobenen Ergebnisse hinaus finden sich aber Aspekte, welche wissenschaftlich weiter zu verfolgen wären:

a. Praxisrelevanz und berufliche Durchsetzungsfähigkeit: Für Absolventen des Verfahrens ist die praktische Hilfe für die Bewältigung des Berufsalltages von hoher Bedeutung. Insbesondere da, wo es um das Durchsetzen eigener Positionen und das Überwinden von Ängsten oder falschen Vorstellungen von beruflichem Ungenügen geht, erleben die Absolventen Gewinn aus der Kompetenzanerkennung. Das ist positiv und überraschend, da dieses Kompetenzanerkennungsverfahren vorrangig auf die Einhaltung von Anerkennungsstandards Wert legt und aus der Einreichung von Nachweisen besteht. Eine erklärende Hypothese ist, dass das dreitägige Assessment-Center, welches jeder durchlaufen muss, hier große Wirkung entfaltet sowie insgesamt das Erfolgserlebnis, ein Zertifikat erreicht zu haben. Möglicherweise wirkt auch die persönliche Begleitung durch eine pädagogische Mitarbeiterin stärkend. 
b. Kritische und affirmative Positionierung zu Disziplin und Bildungssystem sowie zur „Unterstellung “ aus Validierung zu profitieren: Bei einigen induktiv gebildeten Kategorien zeigt sich, dass die Absolventen einerseits begeistert und andererseits kritisch gegenüber Aspekten der Profession und Professionalisierung sind. Das betrifft erworbene Wissensinhalte, Bildungssystem gesamt, Erwachsenenbildungssystem konkret, Pädagogik und Erwachsenenpädagogik als Disziplinen, die Unterstellung durch das Validierungsverfahren zu lernen/zu profitieren. Eine These zur kritischen Sicht ist, dass sich hier auch ein Strang des österreichischen Fach- und Wissenschaftsdiskurses spiegelt, wie er bspw. von der „kritischen Erwachsenenbildung“ (Holzer 2017; Erler et al. 2012) vertreten wird. Es zeigt sich hier die Wirkung der WBA, dass Erwachsenenbildung als Profession Gegenstand der Reflexion von Erwachsenenbildnern wird. Sie befördert die Fähigkeit von Erwachsenenbildnern zur kompetenten Auseinandersetzung mit dem Geschehen rund um ihre eigene Professionalisierung.

\section{Zusammenfassung und Diskussion}

Die WBA wirkt individuell und strukturell professionalisierend. Dort, wo strukturelle Professionalisierung harte Faktoren, wie gehobene positions-, tätigkeits- oder monetär gebundene Möglichkeiten am Arbeitsplatz umfasst (Valorisierung), bewirkt das summative qualifikationsvergebende Verfahren der WBA jedoch wenig. Dies ist nicht dem Validierungsprocedere im engeren Sinne geschuldet. Denn das WBAVerfahren ist, wie bereits weiter oben erwähnt, dem non-formalen Bildungsbereich zugeordnet und sein Erfolg hängt vom Zusammenspiel der relevantesten Akteure aus Bildung, Politik und Arbeitswelt im Bereich ab (,,autonomer Typus“). Insofern geben die Ergebnisse Auskunft darüber, dass dieses Zusammenspiel zu verbessern wäre.

Dort, wo strukturelle Professionalisierung den Rückgriff auf vorhandene Definitionen der Profession, von „ErwachsenenbildnerIn“ und auch von „Erwachsenenbildungslandschaft" bedeutet, ist die WBA äußerst erfolgreich, indem die Absolventen diese Aspekte in ihre individuelle Professionalisierung aufnehmen und sich in hohem Ausmaß damit identifizieren. Letzteres ist hoch bedeutsam, auch mit Blick auf Ergebnisse der Professionalisierungsforschung im deutschsprachigen Raum. Immer wieder wird hier eine zu geringe Identifikation mit der Profession der Erwachsenenbildner bei ihren Angehörigen festgestellt. ${ }^{7}$ Wenn die Identifikation mit der Profession Erwachsenenbildner gesteigert wird, wird auch die Bereitschaft zur Mitwirkung an der Gestaltung derselben gesteigert.

Im Bereich individuell verorteter Professionalität (individuelle Professionalisierung), fördert das Verfahren der WBA in hohem Ausmaß Selbstreflexion und persönliche Weiterentwicklung, also personale Kompetenzen. Die Analyse erlaubt weniger Auskunft darüber, wie die WBA-Absolventen in den Kompetenzbereichen Didaktik, Beratung, Bildungsmanagement profitiert haben, allein im Bereich vermehrte Nutzung digitaler Medien zeigt sich, dass kein individueller Kompetenzzuwachs erzielt

\footnotetext{
${ }^{7}$ Einen Überblick bietet Steiner (2018, S. 163 ff.)
} 
wird. Etwa die Hälfte der Befragten jedoch erwarb zusätzliche Kompetenzen, die am Arbeitsplatz verwendet werden können, welche dies genau sind, müsste in einer weiteren Untersuchung geklärt werden. Auf jeden Fall aber stärkt die WBA die Fähigkeit, sich am Arbeitsplatz durchzusetzen und löst die Wahrnehmung eigener Fähigkeiten und Stärken aus.

Hervorstechend ist bei der qualitativen Inhaltsanalyse, dass die Auseinandersetzung mit vielen inhaltlichen und systembezogenen Aspekten der Profession Erwachsenenbildung angestoßen wird. Damit wird die Selbstreflexionskompetenz, welche für Erwachsenenpädagogen elementar ist (Pachner 2013), gesteigert. Andererseits stößt die WBA Reflexionen über die wissenschaftliche Basis und das „Bildungssystem Erwachsenenbildung“ an. Moniert wird in der Erwachsenenbildungsforschung, dass kaum Mitwirkung der Erwachsenenbildner an ihrer eigenen Professionalisierung vorhanden ist bzw. dass diese nicht gefragt sei (Aschemann und Schmid 2015; vgl. die Darstellung des wissenschaftlichen Diskurses in Steiner 2018, S. $120 \mathrm{ff}$.). Diese Mitwirkung beinhaltet jedenfalls sowohl affirmative als auch kritische Sichtweisen. Genau diese werden durch die WBA ausgelöst. Insgesamt zeigt sich, dass die WBA-Kompetenzvalidierung Professionsangehörige dazu anregt bzw. befähigt, an der Weiterentwicklung der eigenen Profession mitzuwirken. Bezüglich eines Teils der politischen Intentionen in Verbindung mit Validierung von informell und nonformal erworbenen Kompetenzen kann festgestellt werden, dass die WBA eine Steigerung der Motivation für lebensbegleitendes Lernen bewirkt.

Danksagung Für die Unterstützung und Hinweise zur Methodik und Vorgehensweise gilt mein Dank den Begutachtenden dieses Artikels sowie PD Dr. Sascha Sardadvar. Bei WBA-Mitarbeiterin Gudrun Breyer bedanke ich mich herzlich für ihre Hinweise zur Erhebung.

Open Access Dieser Artikel wird unter der Creative Commons Namensnennung 4.0 International Lizenz (http://creativecommons.org/licenses/by/4.0/deed.de) veröffentlicht, welche die Nutzung, Vervielfältigung, Bearbeitung, Verbreitung und Wiedergabe in jeglichem Medium und Format erlaubt, sofern Sie den/die ursprünglichen Autor(en) und die Quelle ordnungsgemäß nennen, einen Link zur Creative Commons Lizenz beifügen und angeben, ob Änderungen vorgenommen wurden.

Publisher's Note Springer Nature remains neutral with regard to jurisdictional claims in published maps and institutional affiliations.

\section{Literatur}

Aschemann, B., \& Schmid, K. (Hrsg.). (2015). Die blinden Flecken im Professionalisierungsdiskurs. Arbeitsrealität in der Erwachsenenbildung. http://www.erwachsenenbildung.at/magazin/15-26/meb1526.pdf. Zugegriffen: 3. Juni 2018. Magazin erwachsenenbildung.at. Das Fachmedium für Forschung, Praxis und Diskurs. Ausgabe 26, 2015.

Biffl, G., Pfeffer, T., \& Skrivanek, I. (2012). Anerkennung ausländischer Qualifikationen und informeller Kompetenzen in Österreich. https://www.donau-uni.ac.at/imperia/md/content/department/ migrationglobalisierung/forschung/biffl-anerkennung-validierung-2012.pdf. Zugegriffen: 12. Mai 2018.

Brünner, A., \& Gruber, E. (2014). Ergebnisse der quantitativen Erhebung 2013 mit Student/inn/en der Weiterbildungsakademie Österreich (WBA). Evaluation des „Kooperativen Systems der österreichischen Erwachsenenbildung“. https://WBA.or.at/media/pdf/WBA-evaluation-endbericht-uni-klagenfurt2013.pdf?m=1518511393\&. Zugegriffen: 14. Mai 2018.

Brünner, A., Gruber, E., \& Huss, S. (2012). Qualifizierung des Weiterbildungspersonals: Ergebnisse aus dem begleitenden Evaluationsprozess der Weiterbildungsakademie Österreich. In E. Gruber \& G. 
Wiesner (Hrsg.), Erwachsenenpädagogische Kompetenzen stärken. Kompetenzbilanzierung für Weiterbildner/-innen (S. 97-116). Bielefeld: W. Bertelsmann.

Bundesministerium für Bildung (2017). Strategie zur Validierung nicht-formalen und informellen Lernens in Österreich. https://bildung.bmbwf.gv.at/euint/eubildung/vnfil.pdf?6fa5oh. Zugegriffen: 3. Juni 2018.

Cedefop (2010). Professional development opportunities for in-company trainers. A compilation of good practices. Luxembourg: Publications Office of the European Union.

Cedefop (2014). Terminology of European Education and Training Policy. Second Edition. A Selection of 130 Key Terms. Luxembourg: Publications Office of the European Union.

Cedefop (2016a). Europäische Leitlinien für die Validierung nicht formalen und informellen Lernens. Luxembourg: Publications Office of the European Union.

Cedefop (2016b). Monitoring the use of validation of non-formal and informal learning. Thematic report for the 2016 update of the European inventory on validation. Luxembourg: Publications Office of the European Union.

Cedefop (2017). European Inventory on validation of non-formal and informal learning - 2016 update. Synthesis report. Luxembourg: Publications Office of the European Union.

Erler, I., Holzer, D., Kloyber, C., \& Ribolits, E. (2012). Kritisch denken: für eine andere Erwachsenenbildung. Schulheft, Bd. 4/12. Innsbruck, Wien, Bozen: Studienverlag.

EUCEN (2013). Validation prize 2013. http://www.observal-net.eu/validation-prize. Zugegriffen: 11. Dez. 2018.

Europäische Union (2012). Empfehlung des Rates vom 20. Dezember 2012 zur Validierung nichtformalen und informellen Lernens. https://eur-lex.europa.eu/legal-content/DE/TXT/?uri=OJ:C:2012:398: TOC. Zugegriffen: 3. Juni 2018. European observatory of validation of nonformal and informal learning.

Gruber, E. (2017). Länderbeispiel Österreich: Die Weiterbildungsakademie (WBA). http://magazin.vhs. or.at/magazin/2017-2/263-winter-201718/schwerpunkt-validierung/laenderbeispiel-oesterreich-dieweiterbildungsakademie-WBA/. Zugegriffen: 12. Mai 2018. Die Österreichische Volkshochschule. Magazin für Erwachsenenbildung. Winter 2017/18, Heft 263/68. Jg., Wien.

Gruber, E. (2018). Kompetenzanerkennung und -zertifizierung für in der Erwachsenenbildung/Weiterbildung Tätige. In R. Tippelt \& A. von Hippel (Hrsg.), Handbuch Erwachsenenbildung/Weiterbildung (Bd. 2, S. 1089-1108). Wiesbaden: Springer.

Gugitscher, K., \& Schmidtke, B. (2018). Kompetenzanerkennung als professioneller Aushandlungsprozess in der Erwachsenenbildung. https://www.die-bonn.de/id/36881/about/html/. Zugegriffen: 11. Dez. 2018. Zeitschrift für Weiterbildungsforschung 2/2018.

Heidemann, W., \& Kruse, W. (1999). „VALID LEONARDO-Projekt“ - Bewertung und Anerkennung von Kompetenzen und Qualifikationen. Europäisches Diskussionspapier für die Sozialpartner. https:// www.econstor.eu/bitstream/10419/116464/1/hbs_arbp_001.pdf. Zugegriffen: 7. Nov. 2018. Arbeitspapier, No. 1, Düsseldorf: Hans-Böckler-Stiftung.

Heilinger, A. (2012). Professionalisierung mit Kompetenz steuern am Beispiel der Weiterbildungsakademie Österreich. In E. Gruber \& G. Wiesner (Hrsg.), Erwachsenenpädagogische Kompetenzen stärken. Kompetenzbilanzierung für Weiterbildner/-innen (S. 59-81). Bielefeld: W. Bertelsmann.

Helsper, W. \& Tippelt, R. (2011). Ende der Profession und Professionalisierung ohne Ende? Zwischenbilanz einer unabgeschlossenen Diskussion. In W. Helsper und R. Tippelt (Hrsg.), Pädagogische Professionalität. Beiheft zur Zeitschrift für Pädagogik, 57 (S. 268-288). Weinheim u.a: Beltz.

Holzer, D. (2017). Weiterbildungswiderstand. Eine kritische Theorie der Verweigerung. Bielefeld: Transcript.

Käpplinger, B. (2002). Anerkennung von Kompetenzen: Definitionen, Kontexte und Praxiserfahrungen in Europa. http://www.die-bonn.de/esprid/dokumente/doc-2002/kaepplinger02_01.pdf. Zugegriffen: 7. Nov. 2018. Bonn: Deutsches Institut für Erwachsenenbildung.

Kraus, K. (2012). Professionalisierung und Beschäftigung in der Weiterbildung. In I. Sgier \& S. Lattke (Hrsg.), Professionalisierungsstrategien der Erwachsenenbildung in Europa. Entwicklungen und Ergebnisse aus Forschungsprojekten (S. 35-46). Bielefeld: W. Bertelsmann.

Mayring, P. (2010). Qualitative Inhaltsanalyse. Grundlagen und Techniken. Weinheim, Basel: Beltz.

Nittel, D. \& Schütz, J. (2013). Zwischen Verberuflichung und Professionalität: Professionalisierungsdynamiken und Anerkennungskämpfe in der sozialen Welt der Erzieherinnen und Weiterbildner. In B. Käpplinger, S. Robak \& S. Schmidt-Lauff (Hrsg.), Engagement für die Erwachsenenbildung. Ethische Bezugnahmen und demokratische Verantwortung (S. 111-130). Wiesbaden: Springer SV.

Pachner, A. (2013). Selbstreflexionskompetenz. Voraussetzung für Lernen und Veränderung in der Erwachsenenbildung? http://www.erwachsenenbildung.at/magazin/13-20/meb13-20.pdf. Zugegriffen: 
11. Dez. 2018. Magazin erwachsenenbildung.at. Das Fachmedium für Forschung, Praxis und Diskurs. Ausgabe 20, 2013.

Prokopp, M., \& Luomi-Messerer, K. (2010). European inventory on validation of non-formal and informal learning 2010. https://WBA.or.at/ueber_uns/CaseStudyRecognitionforprofessionalisationintheadult educationsector.pdf. Zugegriffen: 28. Mai 2018. Case Study: Recognition for Professionalisation in the adult learning sector - Academy of Continuing Education (WBA), Austria.

Schmid, M. (2018). Standards für die Validierung erwachsenenpädagogischer Kompetenzen - kritische Befunde zur Anwendung von quantitativen Gütekriterien bei der Zertifizierung non-formalen und informellen Lernens. https://doi.org/10.1007/s40955-018-0113-9. Zugegriffen: 11. Dez. 2018. Zeitschrift für Weiterbildungsforschung 2/2018, 159-172.

Steiner, P.H. (2018). Soziale Welten der Erwachsenenbildung. Eine professionstheoretische Verortung. Bielefeld: Transcript.

Steiner, M., Wagner, E., \& Pessl, G. (2014). ESF Beschäftigung Österreich 2007-2013. Bereich Erwachsenenbildung. Endbericht 2014 zur Evaluierung im Auftrag des BMBF. https://erwachsenenbildung. at/downloads/service/ESF_Endbericht2014.pdf. Zugegriffen: 12. Mai 2018.

Strauch, A., Jütten, S., \& Mania, E. (2009). Kompetenzerfassung in der Weiterbildung. Instrumente und Methoden situativ anwenden. Bielefeld: W. Bertelsmann.

Weiterbildungsakademie Österreich (2017). Evaluationsbericht. Auswertung der Onlinebefragung anlässlich ,10 Jahre WBA “. Unveröffentlichter Bericht

Weiterbildungsakademie Österreich (2018). Zahlen/Daten/Fakten zur WBA (Stand 27.09.2018). https:// WBA.or.at/de/ueber-uns/daten-und-fakten.php. Zugegriffen: 6. Nov. 2018.

Weiterbildungsakademie Österreich (o.J.). So arbeitet die WBA. https://WBA.or.at/de/fachinfo/soarbeitet-die-WBA.php. Zugegriffen: 3. Dez. 2018.

Werquin, P. (2009). Recognition of non-formal and informal learning in OECD countries: An overview of some key issues. https://doi.org/10.3278/REP0903W011. Zugegriffen: 11. Dez. 2018. REPORT Zeitschrift für Weiterbildungsforschung 03/2009, 11-23. 